\title{
Comparison of LOPES measurements with COREAS and REAS 3.11 simulations
}

Cite as: AIP Conference Proceedings 1535, 84 (2013); https://doi.org/10.1063/1.4807526

Published Online: 23 May 2013

M. Ludwig, W. D. Apel, J. C. Arteaga-Velázquez, et al.

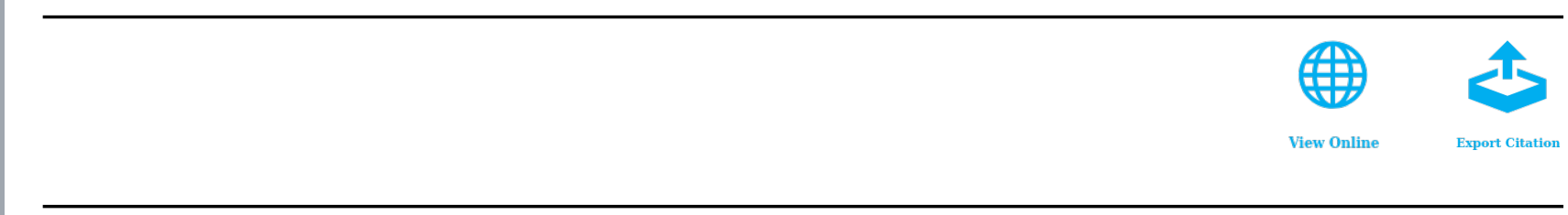

\section{ARTICLES YOU MAY BE INTERESTED IN}

Cosmic ray measurements with LOPES: Status and recent results

AIP Conference Proceedings 1535, 78 (2013); https://doi.org/10.1063/1.4807525

LOPES-3D - vectorial measurements of radio emission from cosmic ray induced air showers AIP Conference Proceedings 1535, 94 (2013); https://doi.org/10.1063/1.4807528

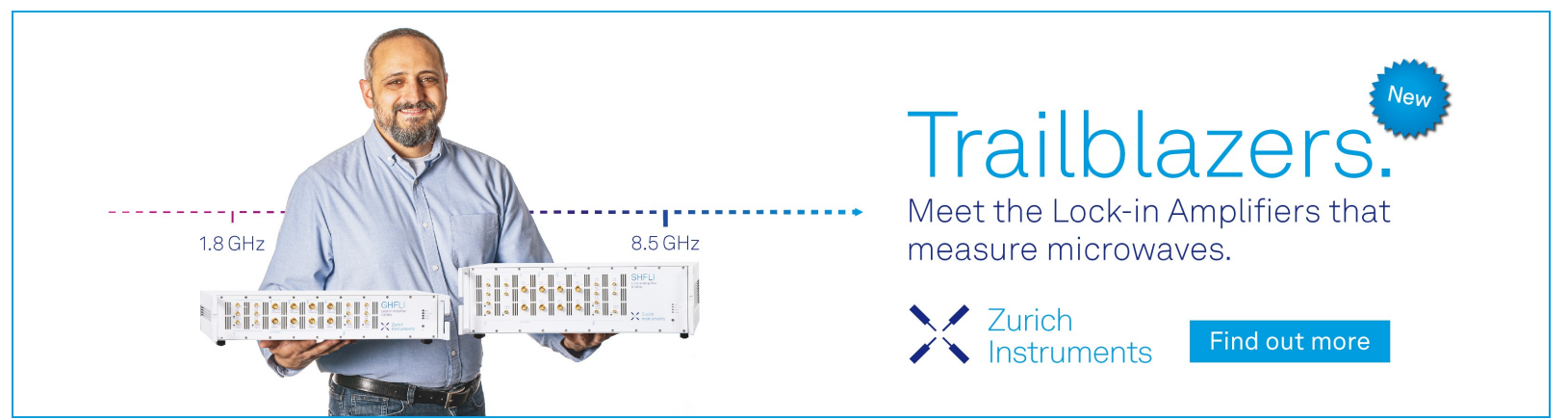




\title{
Comparison of LOPES measurements with CoREAS and REAS 3.11 simulations
}

\author{
M. Ludwig*, W.D. Apel ${ }^{\dagger}$, J.C. Arteaga-Velázquez**, L. Bähren ${ }^{*}$, K. Bekk ${ }^{\dagger}$, \\ M. Bertaina ${ }^{\S}$, P.L. Biermann`, J. Blümer ${ }^{\dagger}, *$, H. Bozdog ${ }^{\dagger}$, I.M. Brancus ${ }^{\|}$, \\ A. Chiavassa ${ }^{\S}$, K. Daumiller ${ }^{\dagger}$, V. de Souza ${ }^{\dagger \dagger}$, F. Di Pierro ${ }^{\S}$, P. Doll ${ }^{\dagger}$, R. Engel ${ }^{\dagger}$,

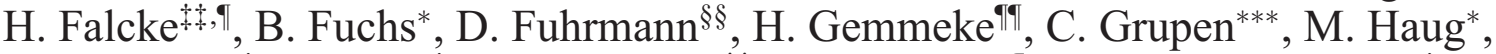 \\ A. Haungs ${ }^{\dagger}$, D. Heck ${ }^{\dagger}$, J.R. Hörandel ${ }^{\dagger \dagger}$, A. Horneffer $₫$, D. Huber*, T. Huege ${ }^{\dagger}$, \\ P.G. Isar ${ }^{\dagger \dagger}$, K.-H. Kampert ${ }^{\S}$, D. Kang*, O. Krömer ${ }^{\uparrow \uparrow}$, J. Kuijpers ${ }^{\dagger \ddagger}$, K. Link*, \\ P. Łuczak $^{+\dagger}$, H.J. Mathes ${ }^{\dagger}$, M. Melissas*, C. Morello ${ }^{\S \S}$, J. Oehlschläger ${ }^{\dagger}$,

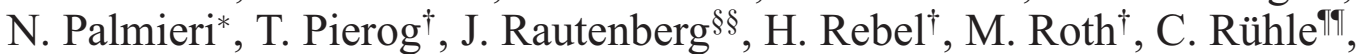

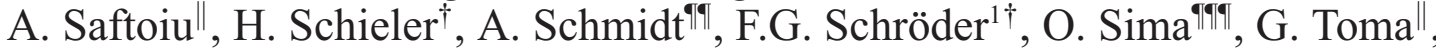

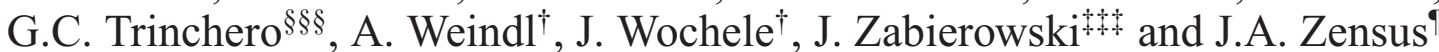

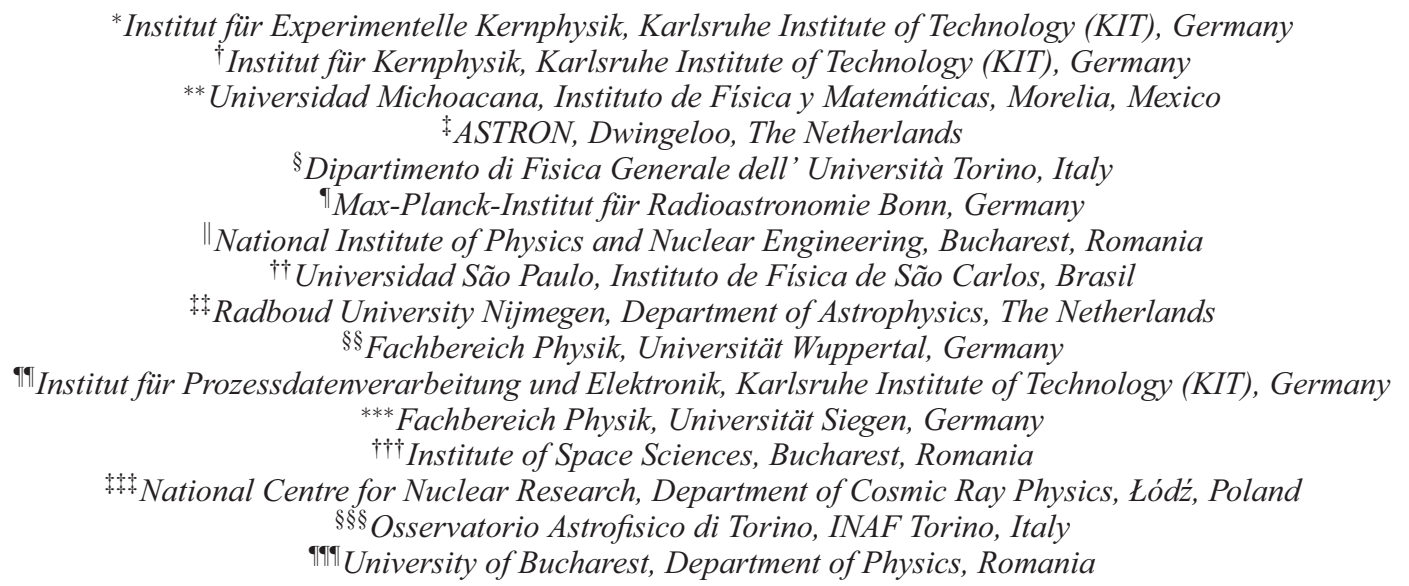

\begin{abstract}
In the previous years, LOPES emerged as a very successful experiment measuring the radio emission from air showers in the MHz frequency range. In parallel, the theoretical description of radio emission was developed further and REAS became a widely used simulation Monte Carlo code. REAS 3 as well as CoREAS are based on the endpoint formalism, i.e. they calculate the emission of the air-shower without assuming specific emission mechanisms. While REAS 3 is based on histograms derived from CORSIKA simulations, CoREAS is directly implemented into CORSIKA without loss of information due to histogramming of the particle distributions. In contrast to the earlier versions of REAS, the newest version REAS 3.11 and CoREAS take into account a realistic atmospheric refractive index. To improve the understanding of the emission processes and judge the quality of the simulations, we compare their predictions with high-quality events measured by LOPES. We present results concerning the lateral distribution measured with 30 east-west aligned LOPES antennas. Only the simulation codes including the refractive index (REAS 3.11 and CoREAS) are able to reproduce the slope of measured lateral distributions, but REAS 3.0 predicts too steep lateral distributions, and does not predict rising lateral distributions as seen in a few LOPES events. Moreover, REAS 3.11 predicts an absolute amplitude compatible with the LOPES measurements.
\end{abstract}

Keywords: radio detection, cosmic rays, air showers, LOPES

PACS: $96.50 . \mathrm{sd}, 95.55 . \mathrm{Jz}$

\section{INTRODUCTION}

${ }^{1}$ Corresponding author

Email address: frank.schroeder@kit.edu
The LOPES experiment $[1,2]$ has made important contributions to the field of radio detection of cosmic ray air

5th International Workshop on Acoustic and Radio EeV Neutrino Detection Activities AIP Conf. Proc. 1535, 84-88 (2013); doi: 10.1063/1.4807526

(C) 2013 AIP Publishing LLC 978-0-7354-1159-3/\$30.00 
showers in the last ten years. With an amplitude and time calibration [3, 4] as well as a careful noise treatment [5], the quality of data reconstruction was improved continuously. To make the radio technique competitive to other detection methods, it is important to understand the radio emission mechanism in detail. To achieve this goal, we compare the lateral distributions measured by LOPES with the predictions of different simulations, i.e. REAS 3.0 [6], REAS 3.11 [7] and CoREAS [8]. For details on the different simulations, please see [9]. All simulation codes are based on the end-point formalism [10] and calculate the radio emission of air-showers simulated with CORSIKA [11]. In contrast to the REAS 3.11 and CoREAS, REAS 3.0 does not include the refractive index of the air, which changes the coherence conditions of the emission $[12,7]$. While CoREAS is implemented directly in CORSIKA, REAS uses the output of CORSIKA simulations, namely histograms of the particle distributions.

For the comparison shown in this article, we analyzed 128 events recorded with the 30 east-west aligned LOPES antennas in the year 2005-2006, which survive high-quality cuts (for details on these cuts, we kindly refer to [13]).

\section{METHOD}

For each LOPES event, we fit an exponential function to the lateral distribution function (LDF) which has been found to be reasonable for the LOPES distance range $[14,15]$. The lateral distribution of the radio signal is the variation of the radio amplitude $\varepsilon$ with the distance to the air shower axis $d_{\text {axis }}$. With the fitting parameters $\eta$ and $\varepsilon_{100}$ the lateral distribution function (LDF) is given by

$$
\varepsilon\left(d_{\text {axis }}\right)=\varepsilon_{100} \cdot \exp \left[-\eta\left(d_{\text {axis }}-100 \mathrm{~m}\right)\right]
$$

where $\eta$ corresponds to the slope of the lateral distribution and $\varepsilon_{100}$ to the electric field at $100 \mathrm{~m}$ lateral distance. Thus, a falling LDF would correspond to $\eta>0$, while a rising LDF corresponds to $\eta<0$.

For all measured events, we get the reconstruction for the cosmic ray particle of KASCADE[16] or KASCADE-Grande[17], i.e. the primary energy, the incoming direction, the core position on ground and the number of electrons and muons measured on ground. The high quality of the reconstruction and the LOPES data gives the opportunity to test models, such as REAS and CoREAS. For each individual event, the KASCADE(-Grande) parameters, namely the energy, incoming direction and core position, are taken as input for the simulation. With CONEX [18], we prepared 200 air shower simulations for proton as primary particle and

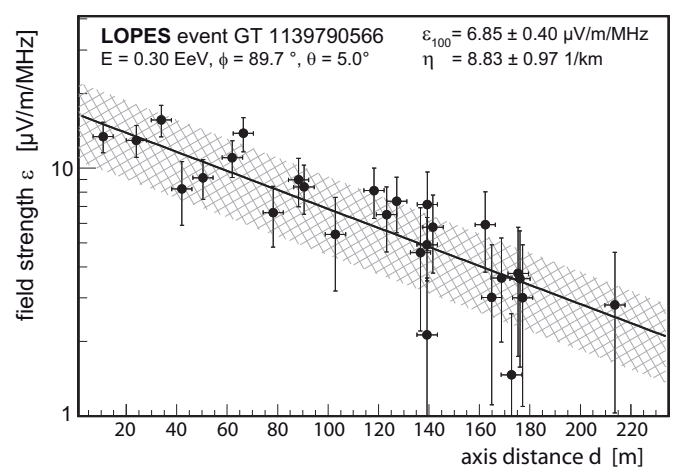

FIGURE 1. Example for a typical lateral distribution measured with LOPES.

100 air shower simulations for an iron nucleus as primary particle for each measured LOPES event. Of these simulations, we selected the air shower with the number of muons on ground most comparable to the number of muons measured by KASCADE(-Grande). The selected air-shower was re-simulated with the full Monte Carlo simulation CORSIKA and additionally the radio emission was calculated by CoREAS and REAS. Consequently, the simulated air shower as well as its radio emission should be comparable to the measured one.

The simulations provide the electric field vector in the time domain. They need to be filtered to the frequency band of LOPES. For this analysis we applied a simple rectangular filter in the effective band of LOPES, i.e. $43-74 \mathrm{MHz}$, but so far no complete detector simulation was made. After filtering, we fitted the same exponential LDF to the simulated east-west signal per antenna as used for the LOPES data. For each event, the proper positions of each individual antenna were taken into account during simulation, i.e. it is possible to compare single lateral distributions. We will show the LDF for a typical as well as for an exotic event and finally make a quantitative comparison of the lateral slope parameter $\eta$. In the individual LDFs the uncertainty band of the data measured with LOPES corresponds to the $35 \%$ calibration scale uncertainty, whereas the simulations have a scale uncertainty (the same for all models) of about $20 \%$ due to the energy uncertainty, since the energy is used as input and it is roughly proportional to the amplitude.

\section{INDIVIDUAL EVENTS}

In Figure 1, the lateral distribution of a typical event is shown. The primary energy of the cosmic ray particle is $E_{p}=3.0 \times 10^{17} \mathrm{eV}$, the zenith angle is $\Theta=5^{\circ}$. The corresponding simulations are shown in Figure 2 for a 


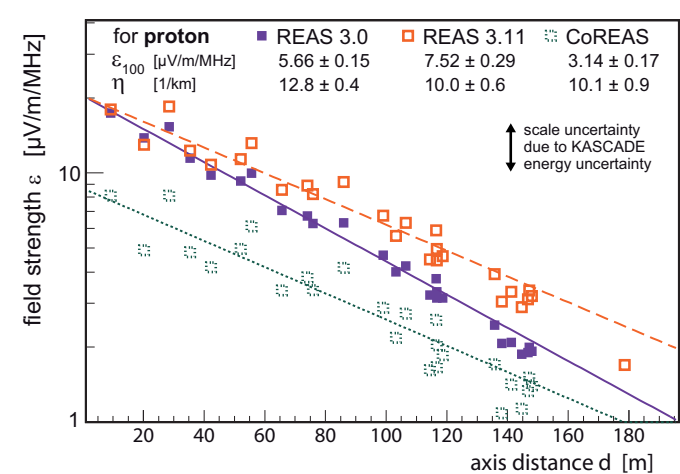

FIGURE 2. Simulations for the event of figure 1, for a proton as primary particle.

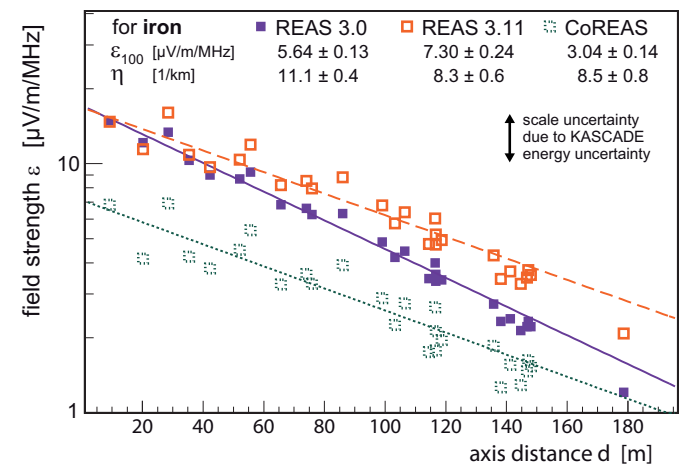

FIGURE 3. Simulations for the event of figure 1, for an iron nucleus as primary particle.

proton as primary particle, and in Figure 3 for an iron nucleus as primary particle.

For REAS 3.0, the slope of the LDF is too steep, i.e. $\eta$ is larger than for the LOPES data, while the predicted $\varepsilon_{100}$ is slightly smaller compared to measurements. For REAS 3.11, the slope parameter as well as the electric field strength are compatible with the measurements within the uncertainties. CoREAS, however, predicts an amplitude smaller than measured with LOPES. On the other hand, the slope parameter of CoREAS agrees with the slope reconstructed from data.

In Figures $4-6$, an example for a non-typical lateral distribution is given. For this event, the cosmic ray particle had a primary energy of $E_{p}=1.1 \times 10^{17} \mathrm{eV}$ and a zenith angle of $\Theta=44^{\circ}$. In the data set of LOPES, there exist a few events like this one which have a rising LDF. With previous versions of existing simulations not including the refractive index of the air, it was not possible to reproduce such structures. REAS 3.0 is one of these simulations which does not predict negative slope parameters.

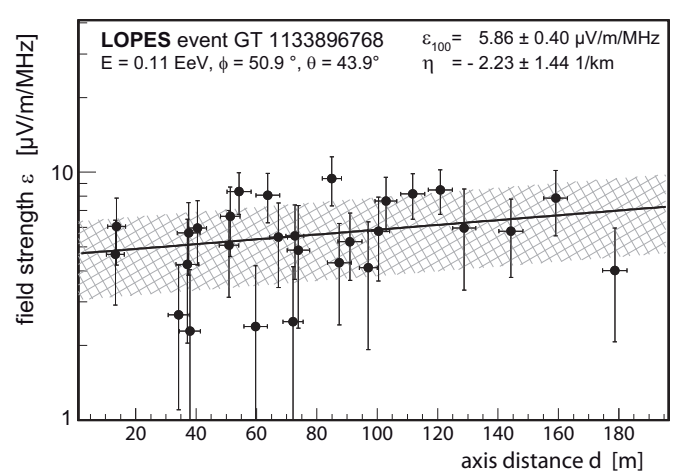

FIGURE 4. Example for a rising lateral distribution measured with LOPES.

In REAS 3.11 and CoREAS, a realistic refractive index of the air is considered, taking into account the variation of the refractive index with the height above ground. Thus, the coherence conditions are influenced due to different light-traveling times which influences the signal strength at individual antenna positions differently. For this event, the slope parameter $\eta$ of REAS 3.11 is negative as well as for the LOPES data, i.e. for the first time, it is possible to reproduce rising LDFs. The predicted amplitude at $100 \mathrm{~m}$ is also compatible within the systematic scale uncertainties. For the predictions derived with CoREAS, the electric field strengths are smaller than the measured amplitudes. Nevertheless, the slope of the LDF is rising for the CoREAS simulations as well.

In the some of the lateral distributions, a slight bending at roughly $120 \mathrm{~m}$ is observed, e.g., in the event shown in Figures 4 to 6 . This coincides with the assumption that a realistic atmospheric index of refraction affects the coherence conditions and thus a "Cherenkov bump" appears $[12,7]$, since the axis distance of $120 \mathrm{~m}$ corresponds roughly to the Cherenkov angle in air. Only at the Cherenkov angle, the radio emission from all stages of the air shower development arrives at approximately the same time and, thus, is amplified.

\section{QUANTITATIVE COMPARISON}

To get a general overview of the agreement between simulations and data, the slope parameter $\eta$ reconstructed for each event is compared. Fig. 7 shows the histograms for LOPES data, Fig. 8 for the proton simulations, and Fig. 9 for the iron simulations. It is obvious that for the REAS 3.0 simulations no negative values of $\eta$ are reconstructed, while in data rising LDFs exist. Also for the falling LDFs, the mean value of the slope parameter is lower for data than for the REAS 3.0 simulations, which consequently cannot describe the LDF measured 


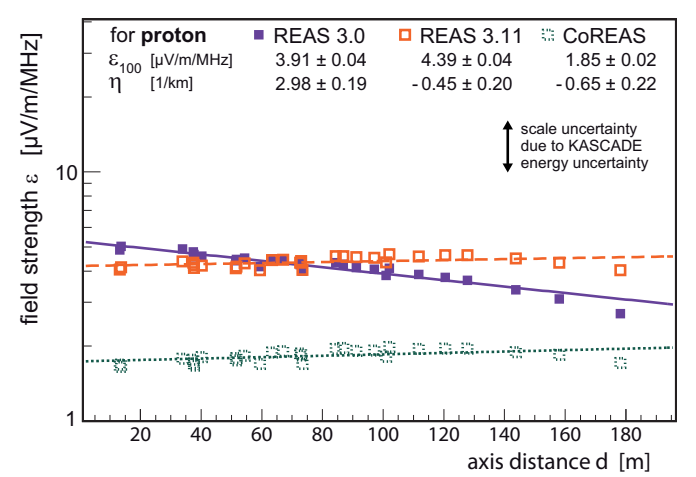

FIGURE 5. Simulations for the event of figure 4, for a proton as primary particle.

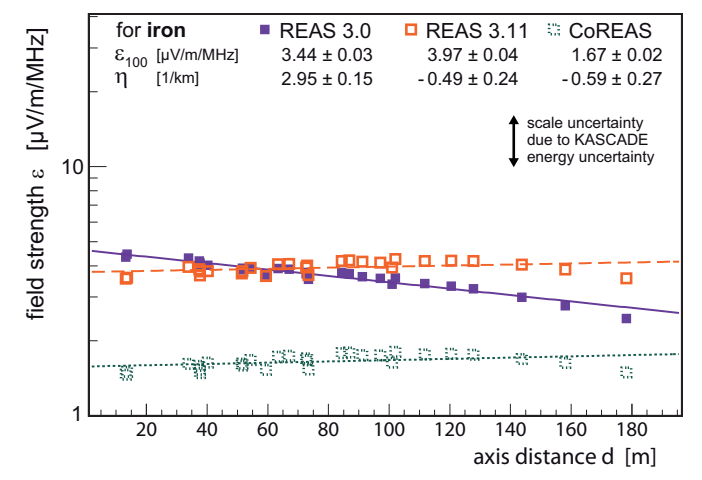

FIGURE 6. Simulations for the event of figure 4, for an iron nucleus as primary particle.

by LOPES properly.

In case of REAS 3.11 and CoREAS, the average slope parameter is close to LOPES measurements, at least for the proton simulations. Even negative values of $\eta$ are reconstructed, i.e. rising LDFs exist in these simulations

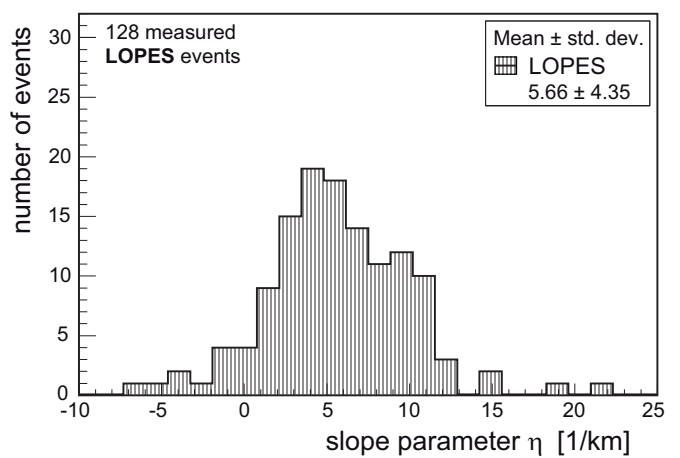

FIGURE 7. Distribution of the slope parameter $\eta$ for measured LOPES events.

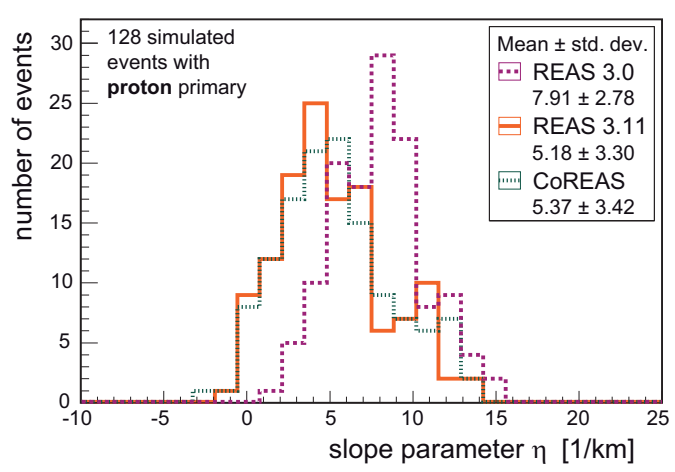

FIGURE 8. Distribution of the slope parameter $\eta$ for simulations with proton as primary particle.

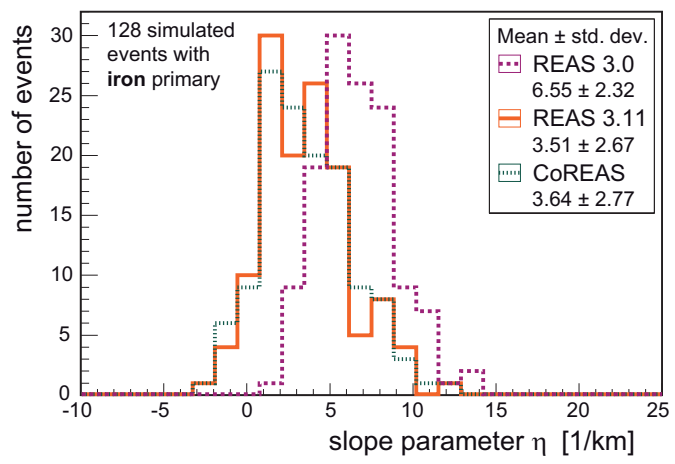

FIGURE 9. Distribution of the slope parameter $\eta$ for simulations with an iron nucleus as primary particle.

as well. No model not taking the refractive index into account could describe this feature of LOPES measurements. Hence, it can be assumed that this feature is related to an atmospheric index which is not unity. This shows the importance of the implementation of a refractive index even for the frequency range of LOPES.

The histograms (Fig. 8 - Fig. 9) show a systematic shift between the average slope of proton and iron simulations. However, the slope will likely be affected by the underlying air-shower simulations, too, e.g., by the used interaction models. Thus, one has to be careful before drawing conclusions on the cosmic ray composition by comparing the LOPES measurements to the simulations. In particular, there might be unknown systematic uncertainties on the scale of $\eta$, which are difficult to estimate by only comparing simulations and radio measurements. For comparison, results from the co-located KASCADEGrande experiments indicate that there is a mixed composition in the energy range considered here, containing a substantial fraction of nuclei heavier than protons [19].

Nevertheless, the fact that there is a difference in the lateral slope of simulated proton and iron showers, fits 
our recent experimental observation that the lateral slope is sensitive to the longitudinal shower development [20], and thus can in principle be used to investigate the composition of the primary cosmic rays. In reference [21], we describe how the lateral slope can be used to reconstruct the atmospheric depth of the shower maximum, $X_{\max }$. This allows us to study the composition with radio measurements in a way similar to air-fluorescence and airCherenkov measurements, but with a much higher dutycycle.

\section{CONCLUSIONS}

The LOPES experiment has been very successful in developing the radio detection method for cosmic ray air showers. Due to the absolute amplitude calibration of LOPES, the data are suited for comparison with the predictions of radio emission simulations on an absolute level. Previously tested simulations could not describe all features of the measured lateral distributions, in particular, they could not describe rising LDFs. With the inclusion of the refractive index in REAS 3.11 and CoREAS, the slope of the measured LDFs are described properly, including rising functions. Furthermore, REAS 3.11 shows a general agreement with LOPES data while CoREAS predicts smaller amplitudes than measured. To understand these discrepancies, the systematic uncertainties on amplitude will be re-investigated and effects of a complete detector simulation on the simulated radio signal will be studied. A detailed analysis of the full LOPES data set including the north-south aligned antennas is under preparation.

\section{ACKNOWLEDGMENTS}

LOPES and KASCADE-Grande have been supported by the German Federal Ministry of Education and Research. KASCADE-Grande is partly supported by the MIUR and INAF of Italy, the Polish Ministry of Science and Higher Education and by the Romanian Authority for Scientific Research UEFISCDI (PNII-IDEI grant 271/2011). This research has been supported by grant number VH-NG413 of the Helmholtz Association.

\section{REFERENCES}

1. H. Falcke et al. - LOPES Collaboration, Nature $\mathbf{4 3 5}$, 313-316 (2005).

2. F.G. Schröder et al. - LOPES Collaboration, "Cosmic Ray Measurements with LOPES: Status and Recent Results," in Proc. of the ARENA 2012 workshop (Erlangen, Germany), AIP Conf. Proc., to be published.
3. S. Nehls, A. Hakenjos, M. J. Arts, et al., Nucl. Instr. and Meth. A 589, $350-361$ (2008).

4. F. Schröder, T. Asch, L. Bähren, et al., Nucl. Instr. and Meth. A 615, 277 - 284 (2010).

5. F.G. Schröder et al. - LOPES Collaboration, Nucl. Instr. and Meth. A (ARENA 2010) 662, Suppl. 1, S238-S241 (2012).

6. M. Ludwig, and T. Huege, Astroparticle Physics 34, 438-446 (2011).

7. M. Ludwig, and T. Huege, Proc. of 32nd ICRC, Beijing, China 2, \#0149 (2011), www.ihep.ac.cn/english/conference/icrc2011/paper.

8. T. Huege, and M. Ludwig, "Simulating radio emission from air showers with CoREAS," in Proc. of the ARENA 2012 workshop (Erlangen, Germany), AIP Conf. Proc., to be published.

9. T. Huege, "Theory and simulations of air shower radio emission," in Proc. of the ARENA 2012 workshop (Erlangen, Germany), AIP Conf. Proc., to be published.

10. C. James, H. Falcke, T. Huege, and M. Ludwig, Phys. Rev. E 84, 056602 (2011).

11. D. Heck, J. Knapp, J. N. Capdevielle, et al., CORSIKA: A Monte Carlo Code to Simulate Extensive Air Showers, FZKA Report 6019, Forschungszentrum Karlsruhe (1998).

12. K. D. de Vries, A. M. van den Berg, O. Scholten, and K. Werner, Phys. Rev. Let. 107, 061101 (2011).

13. F.G. Schröder, $\mathrm{PhD}$ Thesis, Karlsruhe Institute of Technology (KIT), Germany (2011), digbib.ubka.unikarlsruhe.de/volltexte/1000022313.

14. W.D. Apel et al. - LOPES Collaboration, Astroparticle Physics 32, 294-303 (2010).

15. N. Palmieri, $\mathrm{PhD}$ Thesis, Karlsruhe Institute of Technology (KIT), Germany (2012).

16. T. Antoni et al. - KASCADE Collaboration, Nucl. Instr. and Meth. A 513, 490-510 (2003).

17. W.D. Apel et al. - KASCADE-Grande Collaboration, Nucl. Instr. and Meth. A 620, 202-216 (2010).

18. T. Bergmann, et al., Astroparticle Physics 26, 420-432 (2007)

19. W.D. Apel et al. - KASCADE-Grande Collaboration, Phys. Rev. Let. 107, 171104 (2011).

20. W.D. Apel et al. - LOPES Collaboration, Phys. Rev. D 85, 071101(R) (2012)

21. N. Palmieri et al. - LOPES Collaboration, "Reconstructing energy and $X_{\max }$ of cosmic ray air showers using the radio lateral distribution measured with LOPES," in Proc. of the ARENA 2012 workshop (Erlangen, Germany), AIP Conf. Proc., to be published. 